\title{
Can Health Canada protect Canadians from unsafe drugs?
}

$\mathrm{G}$ overnments have promised to modernize Canada's antiquated laws governing therapeutic drugs since 1995 - only to balk on the threshold of change, at best tinkering around its edges. As a consequence, Canadians are left inadequately protected by a federal Food and Drugs Act that's a dusty relic, virtually untouched since 1953. This leaves Health Canada with the Herculean task of ensuring that both old and new medications are as safe as they are effective without the powers, regulatory tools or resources to do so.

Health Canada and CIHR's (Canadian Institutes of Health Research) joint $\$ 30$ million seed investment in a drug safety and effectiveness network is a good start in galvanizing the academic community to mount relevant research. The partnership will be an important step toward more effective safety measures, but it provides only a fraction of the money needed to determine the real risks and comparative benefits of prescription drugs.

The fact is, so little is known about the safety of most novel drug compounds that the releases of new drugs are often essentially experiments - but without a protocol, controls or clear outcomes to measure. The potential consequences include unanticipated deaths and life-threatening complications, as in the widely reported examples of rofecoxib (Vioxx) for arthritis, ${ }^{1}$ rosiglitazone (Avandia) for diabetes ${ }^{2}$ and tegaserod (Zelnorm) for irritable bowel syndrome. ${ }^{3}$ The newly approved oral anticoagulants dabigatran and rivaroxaban should cause comparable concerns given their rapid adoption despite the few studies adequately describing serious potential risks. ${ }^{4}$

At the same time, older drugs are allowed to continue on the market virtually without oversight. Even if concerns about adverse effects are raised under the Food and Drugs Act, Health Canada cannot order postmarket studies to track the experience with a drug. Nor can it monitor a drug company's patient registry or ask a company to conduct systematic reviews of ongoing and completed trials to examine all the data from controlled studies. Health Canada can negotiate a voluntary suspension of sales of a drug or demand withdrawal of an unsafe drug from the market, but — incredibly - requires an exceptionally high degree of certainty before it chooses to force a drug company to comply. Because regulatory authorities wish to respect the autonomy of health professionals, they also attempt to inform and educate physicians, hoping — often in vain — that issuing a so-called "black box warning" to health professionals will curb use. ${ }^{5}$

Now, yet another attempt to modernize our laws is underway, starting with a new round of consultations and testimony from experts. ${ }^{6}$ We have a chance to spur government action, to create a food and drug act that will do everything possible to ensure medications, new and old, are safe and effective.

We believe any reform should start with much more complete evidence being gathered before a drug is approved. For example, pharmaceutical companies could be required to go beyond the limited data of a few randomized trials conducted in selected patients who are most likely to benefit, and also gather evidence from pragmatic randomized trials in large numbers of patients with few restrictions designed to provide real-world estimates of overall benefits and risk.

Adopting this approach, however, would only be feasible if the US Food and Drug Administration and European Medicines Agency also changed their standards. For Health Canada alone to impose stricter laws and regulations would only serve to alienate pharmaceutical companies and potentially damage the Canadian subsidiaries of multinational companies.

Another, perhaps more practical, option would be to do much more monitoring once a drug is released, which is known as progressive drug licensing or a life-cycle approach. ${ }^{7}$ Progressive licensing recognizes that knowledge about risks evolves throughout the life cycle of drugs, because each drug is only a part of a complex therapeutic environment where more than 400 million prescriptions are issued annually. Proponents of progressive licensing argue that it allows more flexibility for regulatory authorities to intervene as risks emerge over time. Opponents argue that this strategy can be used as a justification by pharmaceutical companies to do less premarket testing to get their drug to market faster.

Modernizing our legislation to bring it in line with more up-to-date codes — such as those of the United States and the European Union - is an essential first step to safer drugs. Under a new law, we should substantially strengthen capacity in Canada to conduct large, population-based observational studies of medications, large pragmatic trials and ongoing evaluations of randomized studies using methods such as cumulative meta-analysis.

Important as it is, legislative renewal should only be the first step in a new long-term drug safety strategy for Health Canada. With so few resources, the department might greatly enhance its influence by strengthening partnerships to conduct joint reviews of premarket approval with other major regulators. True partnerships, including a seat at the decisionmaking table, sharing of information and a right of refusals, with large agencies such as the Food and Drug Administration and the European Medicines Agency may achieve greater efficiencies without abandoning Canadian sovereignty.

Another alternative would be to renegotiate cost-recovery strategies with the pharmaceutical industry. Although this approach may get drugs to market more rapidly, it has been hotly contested in the US because it leads to serious conflicts of interest between the industry and regulatory authorities, and potentially results in approval of unsafe drugs that are eventually removed from the market. ${ }^{8}$

Regardless of approach, we should seek economies of scale, efficiencies, greater collaboration among regulatory 
authorities and, ultimately, a much greater focus on postmarketing surveillance.

Under a new law, standards for approval should not be lowered and transparency should be maximal. ${ }^{9}$ Only with strong conviction and political will can Health Canada do a better job of ensuring drugs do more good than harm.

\section{Paul C. Hébert MD MHSc, Matthew B. Stanbrook MD PhD, Noni MacDonald MD MSc, Ken Flegel MDCM MSc, Jane Coutts BA BAA, Stuart MacLeod MD PhD}

\section{References}

1. Kerr DJ, Dunn JA, Langman MJ, et al. Rofecoxib and cardiovascular adverse events in adjuvant treatment of colorectal cancer. $N$ Engl J Med 2007;357:360-369.

2. Nissen SE, Wolski K. Effect of rosiglitazone on the risk of myocardial infarction and death from cardiovascular causes. N Engl J Med 2007;356:2457-71.

3. Wright JM. Progressive drug licensing: an opportunity to achieve transparency and accountability. CMAJ 2007; 176:1848-49.

4. Garcia D, Libby E, Crowthers M. The new oral anticoagulants. Blood 2010;115:15-20.

5. Health Canada. Progressive licensing project. Available: www.hc-sc.gc.ca/dhp -mps/homologation-licensing/plfs_hpfd-eng.php (accessed 2011 Mar. 18).

6. Valiyeva E, Herrmann N, Richon PA, et al. Effect of regulatory warnings on antipsychotic prescription rates among elderly patients with dementia: a populationbased time-series analysis. 2008;179:438-46.
7. Yeates N, Lee DK, Maher M. Health Canada's Progressive Licensing Framework. CMAJ 2007; 176:1845-7.

8. The Institute of Medicine of the National Academies. The future of drug safety: action steps for congress. Washington (DC): The Institute; 2006. Available: www.iom.edu / /media/Files/Report\%20Files/2006/The-Future-of-Drug-Safety/futureofdrugsafety _reportbrief.pdf (accessed 2011 Mar. 18).

9. Hébert PC, Coutts J, Stanbrook MB, et al. Will Open Government make Canada's health agencies more transparent? CMAJ 2011;183:641-2.

Competing interests: See www.cmaj.ca/misc/cmaj_staff.dtl. None declared by Jane Coutts. Stuart MacLeod and his instutition have received funds from Health Canada, and he has served on several expert advisory committees to Health Canada. He has received support from the Canadian Institutes of Health Research, the Canada Foundation for Innovation and the International Development Research Centre.

Affiliations: See www.cmaj.ca/misc/cmaj_staff.dtl. Jane Coutts is an Ottawabased writer and editor and president of Coutts Communicates. Stuart MacLeod is Professor of Pediatrics, University of British Columbia, Vancouver, BC.

Correspondence to: CMAJ editor,pubs@cmaj.ca

Acknowledgement: The authors thank Dr. Robert Peterson for his review of regulatory issues.

Revised 12 July 2011. Previously published at www.cmaj.ca Apr. 18, 2011.

CMAJ 2011. DOI:10.1503/cmaj.110489 